\title{
Arsenic Trioxide Treatment during Pregnancy for Acute Promyelocytic Leukemia in a 22-Year-Old Woman
}

\author{
Claire Cochet $\mathbb{D}^{1},{ }^{1}$ Marion Simonet, ${ }^{2}$ Julie Cattin, ${ }^{1}$ Jean-Patrick Metz, ${ }^{1}$ Ana Berceanu, ${ }^{2}$ \\ Eric Deconinck, ${ }^{2,3}$ Etienne Daguindau, ${ }^{2}$ Françoise Schillinger, ${ }^{4}$ Pierre Fenaux, \\ Nicolas Mottet, ${ }^{1}$ and Yohan Desbrosses ${ }^{2}$ \\ ${ }^{1}$ University Hospital of Besancon, Department of Obstetrics, Besançon F-25000, France \\ ${ }^{2}$ University Hospital of Besancon, Department of Hematology, Besançon F-25000, France \\ ${ }^{3}$ University Bourgogne Franche-Comté, INSERM, EFS BFC, UMR 1098, \\ Interactions Hôte-Greffon Tumeur/Ingénierie Cellulaire et Génique, Besançon F-25000, France \\ ${ }^{4}$ Etablissement Français du Sang Bourgogne Franche Comté, Laboratoire d’hématologie-Immunologie-Biologie Moléculaire, \\ Besançon F-25020, France \\ ${ }^{5}$ Saint Louis Hospital, Paris Diderot University, Paris F-75010, France
}

Correspondence should be addressed to Claire Cochet; claireccht@gmail.com

Received 27 July 2019; Revised 23 September 2019; Accepted 7 February 2020; Published 11 March 2020

Academic Editor: Kazunori Nakase

Copyright (C) 2020 Claire Cochet et al. This is an open access article distributed under the Creative Commons Attribution License, which permits unrestricted use, distribution, and reproduction in any medium, provided the original work is properly cited.

Acute leukemia during pregnancy is rare (1 for 100000 pregnancies). The association of arsenic trioxide (ATO) and all-trans retinoic acid (ATRA) is known as the best therapy in standard-risk acute promyelocytic leukemia (APL). We describe the first case of a pregnancy with ATRA and ATO reported in the literature. In March 2018 at the University Hospital of Besançon, a 22-yearold woman was diagnosed with APL at 14 weeks of gestation (WG). She received a total of $2160 \mathrm{mg}$ of ATRA and $930 \mathrm{mg}$ of ATO between 14 and 35 WG. The mother's cytological remission was very fast. No maternal or fetal complications occurred during pregnancy. The pediatrics outcomes were good. Many case reports about ATRA exposure during the second and third trimesters report no serious adverse effect for pregnancy. ATO is teratogenic, genotoxic, and carcinogenic and passes through the placenta. Fetal exposure seems to be associated with bad pregnancy outcomes (preterm delivery, decreased birth weight, and fetal loss) and with lung diseases in young adults. No clinical trial is obviously possible, and the only data available are environmental exposure or animal studies. This case report may help medical teams to make hard decision for a treatment of APL during pregnancy.

\section{Introduction}

Acute promyelocytic leukemia (APL) is a sub-type of acute myeloid leukemia. It is characterized by a translocation between chromosomes 15 and $17 \mathrm{t}(15: 17)$, which leads to the fusion between the promyelocytic leukemia protein (PML) gene and the retinoic acid receptor $\alpha(\operatorname{RAR} \alpha)$. The association of arsenic trioxide (ATO) and all-trans retinoic acid (ATRA) is known as the best therapy in standard-risk APL compared to the combination of ATRA and anthracyclinebased chemotherapy [1-3].

Acute leukemia during pregnancy is rare (1 for 100000 pregnancies) [4]. APL is curable, but it can have dramatical issues for the mother and the fetus. Its treatment is an emergency. It is associated with ethical questions about the pregnancy outcomes since arsenic is known to be teratogenic. Almost 30 cases of pregnancies with ATRA during the $2^{\text {nd }}$ and/ or $3^{\text {rd }}$ trimester are reported, but none with ATO according to the French teratogen agents reference center (CRAT). We describe the case of a treatment of APL in pregnancy with ATRA and ATO in the University Hospital of Besançon.

\section{Case Description}

In March 2018, the patient, a 22-year-old woman with no medical history, was 14 weeks of gestation (WG). She was 
diagnosed with APL on a blood test, revealing a pancytopenia and a minimal diffuse intravascular coagulopathy (white blood cells (WBC) count $4.2 \times 10^{9} / \mathrm{L}$ with $6 \%$ of circulating hypergranular blasts with bilobed nuclear, neutrophil cells count $2 \times 10^{9} / \mathrm{L}$, hemoglobin rate $11.6 \mathrm{~g} / \mathrm{dL}$, and platelet count $8 \times 10^{9} / \mathrm{L}$ ). Bone marrow aspiration confirmed the diagnosis: $82 \%$ of hypergranular blasts with bundles of Auer rods and a translocation $\mathrm{t}(15 ; 17)$ on cytogenetic analyses (Figure 1). The ratio PML-RAR $\alpha$ was 0.47 .

ATRA $\left(45 \mathrm{mg} / \mathrm{m}^{2} /\right.$ day per os) was introduced immediately after the APL suspicion. The couple was informed about the unknown consequences of the recommended treatment for APL and the absence of malformation risk after $10 \mathrm{WG}$. There was no pregnancy termination demand.

We started a complete induction-treatment and added ATO $0.15 \mathrm{mg} / \mathrm{kg} /$ day with ATRA $\left(45 \mathrm{mg} / \mathrm{m}^{2} /\right.$ day $)$ until the hematological complete remission (Figure 2). Dexamethasone $10 \mathrm{mg}$ twice daily was administered to prevent ATRA differentiation syndrome. Heparin $(50 \mathrm{UI} / \mathrm{kg})$ was also introduced to prevent thrombotic events. The inductiontreatment ended after 4 weeks. The tolerance was good. We quickly obtained a complete cytological remission with minimal residual disease (MRD) (PML-RAR $\alpha=7.4 \times 10^{-4}$ ).

The consolidation-treatment consisted in ATRA $(45 \mathrm{mg} /$ $\mathrm{m}^{2} /$ day) 7 cycles of 2 weeks each 4 weeks and 4 cycles of ATO of 4 weeks each 8 weeks. The MRD became undetectable after the first consolidation-treatment's cycle.

Pregnancy monitoring consisted in monthly ultrasound scans and medical examinations. Sonographies showed no fetal abnormality especially concerning neurological, renal, and cardiac development. Fetus vitality was normal, and its growth reached the $50^{\text {th }}$ percentile. No maternal complication occurred: no bleeding, tension disorder, or preterm birth threat. Clinical examinations and biological observations showed no particularity. No additional treatment was necessary. The hemoglobin rate remained $>10 \mathrm{~g} / \mathrm{dL}$ during the pregnancy.

Since the patient's biological response was total, the consolidation-treatment was suspended after 2 cycles (around $35 \mathrm{WG}$ ) in anticipation of the delivery, to avoid perinatal fetal exposure of arsenic. Pregnancy progress was still totally normal, and no induction of labor was necessary.

At $40+6 \mathrm{WG}$, our patient gave birth to a healthy girl of $3330 \mathrm{~g}$ with an Apgar score of 10 at 5 minutes. The delivery process was normal. The blood sample was proceeded on the new born cord to monitor the hepatic and renal functions. Two electrocardiograms and two blood samples at day 1 and day 4 did not show any cardiac or electrolytic complication. Breast feeding was forbidden.

The consolidation-treatment was resumed 2 months after the delivery.

After the complete treatment, the cytological remission was complete with an undetectable MRD. The patient confessed she did not follow the medical prescription and never took the ATRA during the prenatal consolidationtreatment phase. The patient received a total of $2160 \mathrm{mg}$ of ATRA and $930 \mathrm{mg}$ of ATO while she was pregnant.

The pediatric control 3 months and 9 months after birth showed no complication. The baby's growth and development were normal.

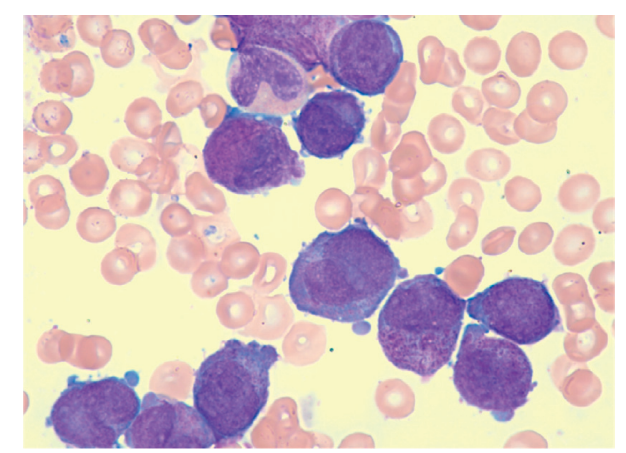

FIgURE 1: Medullary smear: presence of $82 \%$ of hypergranular blasts with bundles of Auer rods.

\section{Results and Discussion}

Cancers during pregnancy represent clinical and ethical dilemmas for medical teams. So far, evidences on pediatric outcomes after most of maternal cancer's treatment during pregnancy are reassuring [5]. APL is an oncologic emergency known to increase the risk of abortion, perinatal mortality, preterm delivery, and intrauterine growth restriction during pregnancy [6].

The combination of ATO and ATRA without chemotherapy significantly improved survival and relapse risk in patients with newly diagnosed standard-risk APL [1-3]. The French-Belgian-Swiss APL group proved in APL 2006 trial that ATO, added to a "classical" ATRA and anthracyclinebased chemotherapy regimen, reduces the incidence of relapse in standard-risk APL [7]. Since the superiority of this treatment is proved, medical teams will have to deal with the question of arsenic use in pregnant women with APL. Our case confirms ATO's relevance since the patient confessed she did not take her ATRA-based treatment during the consolidation phase.

ATRA is teratogenic when given during organogenesis. When used during the $2^{\text {nd }}$ or $3^{\text {rd }}$ trimester of pregnancy in human, fetal arrhythmia and retarded-fetal growth have been described [8]. In our case, there was no teratogenic risk since the treatment began after the organogenesis period (4-10 WG). Many cases of pregnancies with ATRA during the second and third trimesters are reported in the literature with no serious adverse outcomes $[6,9-11]$.

ATO is teratogenic, genotoxic, and carcinogenic. No case report on ATO's use during pregnancy in human are available in the literature, but it passes through the placenta [12]. Animal studies proved the carcinogenic effect of high doses of arsenic (up to $10 \mathrm{mg} / \mathrm{kG} /$ day) $[12,13]$. There is a variation of sensibility to arsenic exposure due to the rate of methylation, which is very different between species. Human sensibility is still unknown [14]. The only data available on intrauterine arsenic exposure effects are environmental studies. It seems to be associated with malignant and nonmalignant lung diseases in young adults through epigenetic effects [15]. Studies in Bangladesh and Taiwan showed that arsenic environmental fetal exposure is associated with decreased birth weight and preterm delivery $[16,17]$. A Bangladesh cohort study also showed that there is 


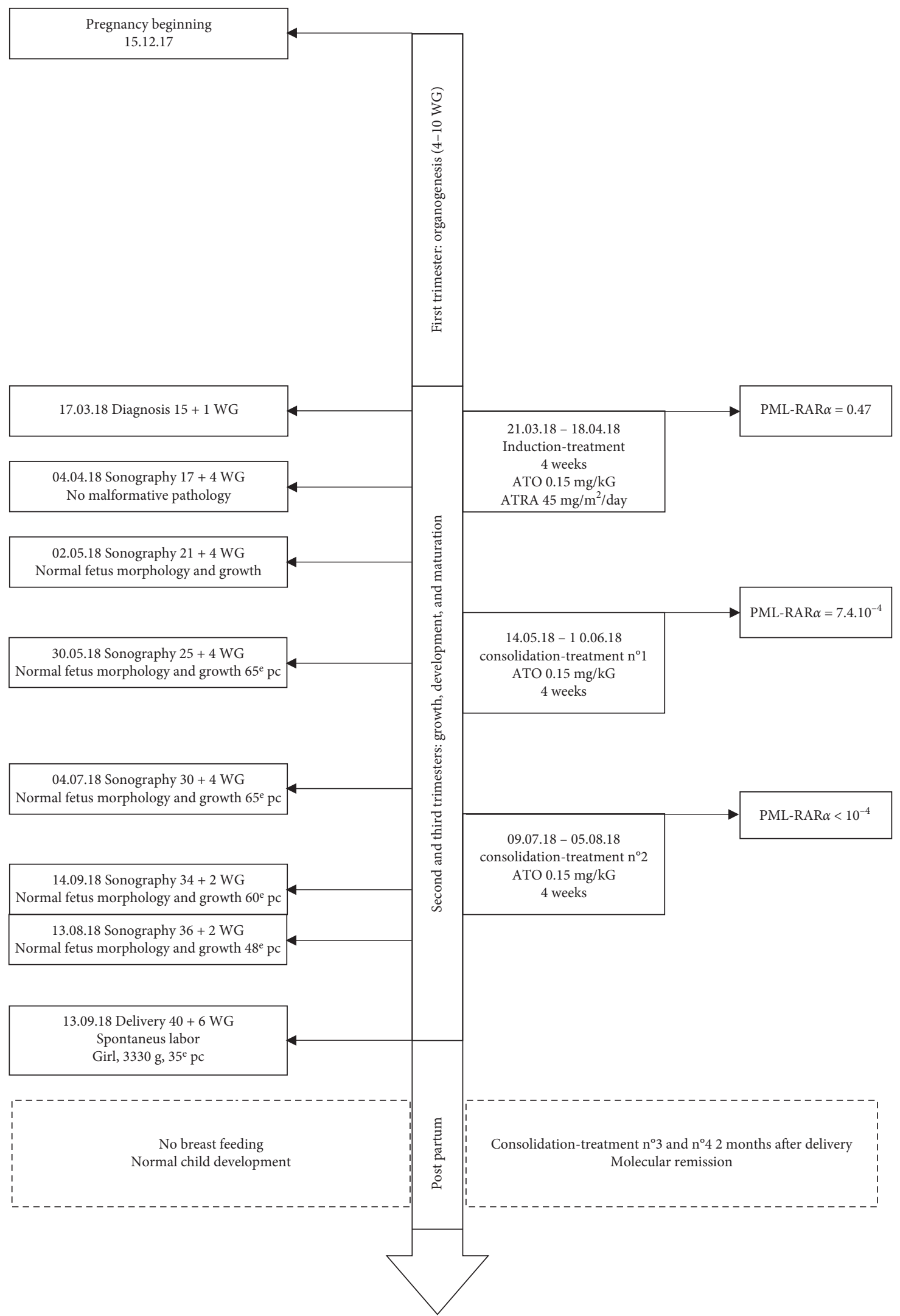

FIgURE 2: Chronology of treatment received, assessment of disease response, and pregnancy control. 
a bigger risk of fetal loss and infant death for women who drink water with more than 50 microgram of arsenic per liter. It also seems to be a dose effect [16]. All those studies are observational and retrospective. The exact dose of arsenic maternal exposure is difficult to weight. Moreover, the environmental exposure also affects the periconceptional period and first trimester of pregnancy. In our case, the patient received more than $900 \mathrm{mg}$ of arsenic trioxide. It is far more than in environmental studies. However, this exposure was limited to the second and third trimesters of pregnancy with a therapeutic window for the delivery. Since no clinical trials are obviously possible on pregnant women, the generalization of our case is difficult.

Regarding the treatment choice, it is important to highlight that any delay can compromise the remission in APL. At time of diagnosis, a medical termination of pregnancy was impossible because our patient had cytopenia and a minimal diffuse intravascular coagulopathy. Despite the limited clinical experience, ATRA was introduced immediately. This treatment seemed reasonably safe when used in patients with APL in second and third trimesters of pregnancy. Indeed, recommendations from the European LeukemiaNet published in 2009 recommend the use of ATRA and chemotherapy in these patients and an induction of labor between cycles of chemotherapy [18]. The update of these recommendations in 2019 did not make any change in the management of APL during pregnancy [19]. Anthracycline-based chemotherapy can increase the risk of abortion, prematurity, low weight, neonatal neutropenia, and sepsis [18]. For these reasons, we were reluctant to use chemotherapy during pregnancy. Moreover, anthracyclinebased chemotherapy induces a long aplasia which can cause infections and hemorrhagic complications. The expected response rate with ATRA alone is not significantly different from ATRA plus chemotherapy in terms of complete remission rate, but it can have unfavorable impact on the risk of relapse [18]. Therefore, after a multidisciplinary consultation, we decided to use ATRA and ATO treatment with stringent fetal monitoring.

In our case, no adverse fetal outcome occurred. The mother's response was very fast, and it seems that pregnancy did not have effect on treatment efficiency. It will take time to assess the long-term effect on the child development of prenatal ATO exposure. Nevertheless, this unique case report may help medical teams to make hard decision for treatment of acute promyelocytic leukemia during pregnancy, whose best option is nowadays known to be ATRA and ATO.

\section{Consent}

The patient gave her consent for the publication of her medical information.

\section{Conflicts of Interest}

The authors declare that they have no conflicts of interest.

\section{Authors' Contributions}

C. C. and M. S. wrote the manuscript and prepared the figures; JP. M., A. B., and J. C. provided helpful advises with the manuscript; F. S. provided additional information and medullary smear picture; P. F., Y. D., E. D., and N. M. reviewed the manuscript.

\section{Acknowledgments}

The authors thank their colleagues of Hematology and Obstetrics Department of University Hospital of Besançon for collaboration on the care of this patient.

\section{References}

[1] F. Lo-Coco, G. Avvisati, M. Vignetti et al., "Retinoic acid and arsenic trioxide for acute promyelocytic leukemia," New England Journal of Medicine, vol. 369, no. 2, pp. 111-121, 2013.

[2] U. Platzbecker, G. Avvisati, L. Cicconi et al., "Improved outcomes with retinoic acid and arsenic trioxide compared with retinoic acid and chemotherapy in non-high-risk acute promyelocytic leukemia: final results of the randomized Italian-German APL0406 trial," Journal of Clinical Oncology, vol. 35, no. 6, pp. 605-612, 2017.

[3] A. K. Burnett, N. H. Russell, R. K. Hills et al., "Arsenic trioxide and all-trans retinoic acid treatment for acute promyelocytic leukaemia in all risk groups (AML17): results of a randomised, controlled, phase 3 trial," The Lancet Oncology, vol. 16, no. 13, pp. 1295-1305, 2015.

[4] U. Abadi, G. Koren, and M. Lishner, "Leukemia and lymphoma in pregnancy," Hematology/Oncology Clinics of North America, vol. 25, no. 2, pp. 277-291, 2011.

[5] T. Vandenbroucke, M. Verheecke, M. Fumagalli, C. Lok, and F. Amant, "Effects of cancer treatment during pregnancy on fetal and child development," The Lancet Child \& Adolescent Health, vol. 1, no. 4, pp. 302-310, 2017.

[6] Y. Chelghoum, N. Vey, E. Raffoux et al., "Acute leukemia during pregnancy," Cancer, vol. 104, no. 1, pp. 110-117, 2005.

[7] L. Adès, X. Thomas, A. G. Bresler et al., "Arsenic trioxide is required in the treatment of newly diagnosed acute promyelocytic leukemia. Analysis of a randomized trial (APL 2006) by the French Belgian Swiss APL group," Haematologica, vol. 103, no. 12, pp. 2033-2039, 2018.

[8] Y. Terada, T. Shindo, A. Endoh, M. Watanabe, T. Fukaya, and A. Yajima, "Fetal arrhythmia during treatment of pregnancyassociated acute promtelocytic leukemia with all-trans retinoic acid and favorable outcome," Leukemia, vol. 11, no. 3, pp. 454-455, 1997.

[9] S. Valappil, M. Kurkar, and R. Howell, "Outcome of pregnancy in women treated with all-trans retinoic acid; a case report and review of literature," Hematology, vol. 12, no. 5, pp. 415-418, 2007.

[10] D. Yang and L. Hladnik, "Treatment of acute promyelocytic leukemia during pregnancy," Pharmacotherapy, vol. 29, no. 6, pp. 709-724, 2009.

[11] K. Agarwal, M. Patel, and V. Agarwal, "A complicated case of acute promyelocytic leukemia in the second trimester of pregnancy successfully treated with all-trans-retinoic acid," Case Reports in Hematology, vol. 2015, Article ID 634252, 4 pages, 2015. 
[12] Report NRC (US) S to U the 1999, A in DW. Experimental Studies, National Academies Press, Washington, DC, USA, 2001.

[13] M. P. Waalkes, J. M. Ward, and B. A. Diwan, "Induction of tumors of the liver, lung, ovary and adrenal in adult mice after brief maternal gestational exposure to inorganic arsenic: promotional effects of postnatal phorbol ester exposure on hepatic and pulmonary, but not dermal cancers," Carcinogenesis, vol. 25, no. 1, pp. 133-141, 2004.

[14] M. Vahter, "Methylation of inorganic arsenic in different mammalian species and population groups," Science Progress, vol. 82, no. Pt 1, pp. 69-88, 1999.

[15] M. Vahter, "Health effects of early life exposure to arsenic," Basic \& Clinical Pharmacology \& Toxicology, vol. 102, no. 2, pp. 204-211, 2008.

[16] K. L. Huyck, M. L. Kile, G. Mahiuddin et al., "Maternal arsenic exposure associated with low birth weight in Bangladesh," Journal of Occupational and Environmental Medicine, vol. 49, no. 10, pp. 1097-1104, 2007.

[17] C.-Y. Yang, C.-C. Chang, S.-S. Tsai, H.-Y. Chuang, C.-K. Ho, and T.-N. Wu, "Arsenic in drinking water and adverse pregnancy outcome in an arseniasis-endemic area in northeastern Taiwan," Environmental Research, vol. 91, no. 1, pp. 29-34, 2003.

[18] M. A. Sanz, D. Grimwade, M. S. Tallman et al., "Management of acute promyelocytic leukemia: recommendations from an expert panel on behalf of the European LeukemiaNet," Blood, vol. 113, no. 9, pp. 1875-1891, 2009.

[19] M. A. Sanz, P. Fenaux, M. S. Tallman et al., "Management of acute promyelocytic leukemia: updated recommendations from an expert panel of the European LeukemiaNet," Blood, vol. 133, no. 15, pp. 1630-1643, 2019. 